Management international

International Management

Gestiòn Internacional

Vers une appréhension plus circonscrite des défis inhérents à la participation des acteurs de la société civile à l'institutionnalisation de la responsabilité sociale de l'entreprise (RSE)

Towards A More Focused Understanding Of The Challenges Inherent In The Participation Of Civil Society To The Institutionalization Of CSR

Hacia una comprensión circunscripta de los desafíos inherentes de la participación de los actores de la sociedad civil en la institucionalización de la responsabilidad social empresarial

Emmanuelle Champion

Volume 20, numéro 3, printemps 2016

URI : https://id.erudit.org/iderudit/1051304ar

DOI : https://doi.org/10.7202/1051304ar

\section{Aller au sommaire du numéro}

Éditeur(s)

HEC Montréal

Université Paris Dauphine

ISSN

1206-1697 (imprimé)

1918-9222 (numérique)

Découvrir la revue

Citer cet article

Champion, E. (2016). Vers une appréhension plus circonscrite des défis

inhérents à la participation des acteurs de la société civile à

l'institutionnalisation de la responsabilité sociale de l'entreprise (RSE).

Management international / International Management / Gestiòn Internacional,

20(3), 27-36. https://doi.org/10.7202/1051304ar

\section{Résumé de l'article}

Cet article propose dix hypothèses de recherche se rapportant aux défis que représentent le déploiement et l'expérimentation par une coalition multi acteurs de modalités d'action inusitées s'inscrivant dans la sphère institutionnelle, tandis qu'elle cherche à établir un rapport de force structurant le champ de régulation de la RSE. Pour ce faire, nous élaborons un cadre d'analyse, à partir d'une hybridation originale entre le courant de l'entrepreneuriat institutionnel et les approches des processus politiques et du cadrage, pour concevoir l'action d'une coalition réunissant des acteurs de la société civile engagés dans une lutte portant sur les modalités d'institutionnalisation de la RSE.
Tous droits réservés @ Management international / International Management / Gestión Internacional, 2016
Ce document est protégé par la loi sur le droit d'auteur. L’utilisation des services d’Érudit (y compris la reproduction) est assujettie à sa politique d'utilisation que vous pouvez consulter en ligne. 


\title{
Vers une appréhension plus circonscrite des défis inhérents à la participation des acteurs de la société civile à l'institutionnalisation de la responsabilité sociale de l'entreprise (RSE) ${ }^{*}$
}

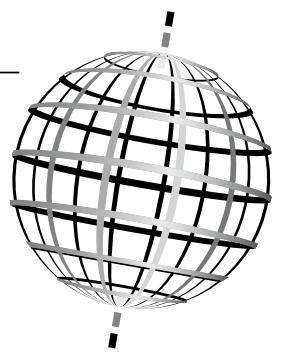

\section{Towards A More Focused Understanding Of The Challenges Inherent In The Participation Of Civil Society To The Institutionalization Of CSR}

\section{Hacia una comprensión circunscripta de los desafíos inherentes de la participación de los actores de la sociedad civil en la institucionalización de la responsabilidad social empresarial}

\author{
EMMANUELLE CHAMPION
}

CRIMT - HEC ${ }^{1}$

\section{RÉSUMÉ}

Cet article propose dix hypothèses de recherche se rapportant aux défis que représentent le déploiement et lexpérimentation par une coalition multi acteurs de modalités d'action inusitées s'inscrivant dans la sphère institutionnelle, tandis qu'elle cherche à établir un rapport de force structurant le champ de régulation de la RSE. Pour ce faire, nous élaborons un cadre d'analyse, à partir d'une hybridation originale entre le courant de l'entrepreneuriat institutionnel et les approches des processus politiques et du cadrage, pour concevoir l'action d'une coalition réunissant des acteurs de la société civile engagés dans une lutte portant sur les modalités d'institutionnalisation de la RSE. Mots clés : responsabilité sociale de l'entreprise, corégulation, coalitions multi acteurs, entreprenariat institutionnel, cadrage culturel, stratégies de diffusion

\section{ABSTRACT}

This article presents ten research hypotheses on the challenges facing a multistakeholder coalition involved in the institutional deployment and experimentation of new modes of action when seeking to strategically define its field of CSR intervention. To this end and on the basis of an original merging of theoretical and methodological approaches (namely those of institutional entrepreneurship, political processes, and cultural framing) we develop an analytical framework for a better apprehension of this coalition's fight over the practical institutionalisation of CSR.

Keywords: corporate social responsibility, coregulation, multi-stakeholder coalitions, Institutional Entrepreneurship, cultural framework, diffusion Strategies

\section{RESUMEN}

Este artículo propone diez hipótesis de investigación que están relacionadas con los desafíos que representan la puesta en marcha y la experimentación, por una coalición de actores múltiples, de diferentes modos de acción inusitados y que se inscriben en una esfera institucional que busca establecer una relación de fuerzas que darán estructura al campo de regulación de la RSE. Para lograr nuestro objetivo, vamos a elaborar un marco de análisis híbrido original que retomara la corriente del emprendimiento corporativo institucional y los enfoques de los procesos políticos y del encuadre, para concebir la acción de una coalición que reúne actores de la sociedad comprometidos con una lucha que facilitará las modalidades de la institucionalización de la RSE.

Palabras Claves: responsabilidad social empresarial, regulación conjunta, coalición de actores múltiples, emprendimiento corporativo institucional, encuadre cultural, estrategias de difusión.
T e champ de régulation de la responsabilité sociale de Ll'entreprise (RSE), dont l'essor réside dans les mécanismes mis de l'avant dans les années quatre-vingt-dix par les entreprises transnationales afin d'assurer leur autonomie législative à léchelle internationale (Gendron et al., 2004), s'articule de plus en plus sur des initiatives qui intègrent de nouveaux acteurs l'orientant ainsi vers un système que l'on qualifie parfois de « régulation civile» (Utting, 2001), de "cadre régulatoire hybride» (Audet, 2013), de « régulation non gouvernementale» (O’Rourke, 2003) ou encore, de «corégulation» (Igalens, 2013). Bien qu'encore marginales, ces initiatives associent à divers degrés, lorsqu'ils n'en sont pas eux-mêmes les instigateurs, des représentants de syndicats, d'Organisations non gouvernementales (ONG), d’organismes communautaires, de groupes environnementaux

1. Centre de recherche interuniversitaire sur la mondialisation et le travail

* Cet article découle d'une thèse de doctorat en administration réalisée sous la direction de Madame Corinne Gendron (UQAM) et Monsieur Christian Lévesque (HEC Montréal) que je remercie très sincèrement pour la qualité de leur encadrement. 
et de mouvements étudiants, pour définir le contenu, voire assurer la mise en œuvre d'instruments de régulation qui peuvent prendre la forme de codes de conduite et de politiques d'approvisionnement responsable, et dont la spécificité est d'insuffler une contrainte normative au sein des chaines globales de valeur. Si plusieurs organisations de la société civile souhaitent prendre part à lédification de ce système de régulation, celles-ci ne mesurent souvent pas adéquatement les difficultés quelles pourraient rencontrer dans ce processus. D’autant que le principal mode opératoire des mouvements sociaux consistait jusqu'alors à s'adresser aux gouvernements pour qu'ils procèdent à des reformes de leur cadre institutionnel.

Dans cet article, nous souhaitons précisément appréhender les défis inhérents à la participation directe d'acteurs de la société civile au processus d'institutionnalisation du champ de régulation de la RSE. Pour ce faire, nous proposons un cadre d'analyse novateur dont la particularité est de combiner la théorie néo-institutionnelle, en particulier le courant de l'entreprenariat institutionnel, et la théorie des mouvements sociaux, notamment les approches des processus politiques et du cadrage, afin de situer leurs actions au cœur des micro-dynamiques à la base du changement institutionnel. Dans un premier temps, nous discuterons de la nécessité de renouveler notre manière de concevoir les relations entre les institutions et les acteurs pour étudier la participation directe de la société civile à la régulation sociale à lère de la mondialisation productive. Puis, nous présenterons un cadre d'analyse pour concevoir les actions que mettent en œuvre les acteurs au sein de ce que nous considérons comme un processus d'expérimentation d'une institution à travers lequel ils se construisent mutuellement (Kristensen et Morgan, 2012; Fligstein et McAdam, 2012). En nous basant sur une littérature essentiellement théorique, nous formulerons plusieurs hypothèses de recherche relativement aux tensions que rencontrent les acteurs de la société civile lorsqu'ils s'engagent dans une lutte portant sur les modalités d'institutionnalisation de la RSE.

\section{Refondre nos cadres d'analyse pour étudier la participation directe de la société civile à la régulation sociale à l'ère de la mondialisation productive}

Plusieurs éléments nous incitent à développer un cadre d'analyse combinant différents champs théoriques, afin détudier la participation directe de la société civile à la régulation sociale à lère de la mondialisation productive ${ }^{2}$. Tandis que les mouvements sociaux s'adressaient durant la période fordiste principalement aux États pour qu'ils transforment en fonction de leurs revendications les cadres institutionnels, force est de constater qu'ils se retrouvent à présent directement impliqués au sein de processus institutionnels inusités, que ce soit à travers leur participation à des initiatives multipartites se consacrant à la production de normes sociales et environnementales, le développement de pratiques commerciales alternatives tels que le commerce équitable et biologique, léconomie sociale et solidaire, la finance socialement responsable etc. Si plusieurs sociologues des mouvements sociaux décèlent une rupture dans leur mode opératoire et proposent un certain nombre de concepts pour témoigner de cette reconfiguration des formes de l'action collective (Gendron, 2001; Michelleti, 2003), ce champ d'étude ne rend cependant pas compte de la complexité des processus institutionnels dans lesquels les acteurs sont engagés, ni des stratégies qu'ils mettent en œuvre pour les maîtriser. En effet, ce courant sociologique s'intéresse davantage aux formes de mobilisations collectives que les nouveaux mouvements sociaux développent en marge de la sphère institutionnelle pour influencer les politiques gouvernementales et le système en général (McAdam et Scott, 2005).

Or, la transformation des cadres institutionnels dans lesquels s'inscrivait l'action des acteurs collectifs nous oblige, depuis notre entrée dans lère post fordiste, à opérer à un renouvèlement de notre manière de concevoir les relations entre les institutions et les acteurs. Si la période fordiste se caractérisait par des configurations institutionnelles négociées et établies en amont par l'État, se complétant à différents niveaux de mise en œuvre, et conférant des identités et des rôles organisationnels prédéfinis et stables aux acteurs collectifs, celles émergeantes apparaissent, au contraire, plus fluctuantes et incertaines (Kristensen et Morgan, 2012). Les cadres institutionnels sétant délités à la faveur d'un système marqué par une pluralité de forces politiques et de centres de décision, les acteurs sociaux se retrouvent plus libres de participer à la construction de nouveaux foyers de régulation qui foisonnent en marge des traditionnels canaux politiques (Beck, 1986). Ils se retrouvent ainsi directement impliqués au sein de processus d'institutionnalisation de mécanismes de régulation, dont la vocation est de transcender les limites territoriales du droit, et à travers lesquels ils sont appelés à se définir des rôles et des identités organisationnels.

Le constat qui sest progressivement imposé sur l'incapacité de notre système de régulation sociale hérité de la période fordiste à encadrer une firme-réseau multinationale, fait aujourd'hui place à l'expérimentation d'instruments de régulation dont la singularité est d'inscrire une contrainte normative au sein des chaînes globales de valeur (Sobczak, 2004). Si les premiers instruments de RSE mis en œuvre dans les années quatre-vingt-dix par les entreprises s'apparentaient à des mécanismes unilatéraux, ils prennent des formes plus hétérogènes (bilatérales et multilatérales), à partir des années deux-milles, à mesure que de nouveaux acteurs de la société civile s'associent à leur développement, voire qu'ils en deviennent eux-mêmes les instigateurs (Kolk et Tolder, 2005). S'inscrivant dans une tendance générale qui participe à lélargissement des acteurs associés à la production

2. Par «mondialisation productive», nous entendons l’organisation par les entreprises transnationales de leurs activités de production sous la forme de réseaux transnationaux au sein desquelles elles agissent comme des donneurs d'ordre, c'est-à-dire qu'elles pilotent l'activité productive entre une multitude de producteurs localisés dans plusieurs pays pour se consacrer exclusivement au contrôle de l'information critique, des compétences et des ressources afin que la chaîne globale de valeur (CGV) fonctionne efficacement (Gereffi, 2001). 
de la régulation (Beck, 1986), on voit progressivement se structurer, à partir de ces initiatives, les bases d'un système que nous considérons comme de la corégulation. Dans ce système, les rapprochements qui sétablissent entre les acteurs de la société civile, sont considérés comme la pierre-angulaire de son effectivité puisque ces instruments, pour le moment dépourvus d'effets juridiquement contraignants, reposent sur les dynamiques sociales qu'ils créent sur le terrain pour assurer leur mise en œuvre (Sobczak, 2008).

Ces nouvelles conditions devraient bouleverser la manière dont nous concevons nos cadres d'analyse puisqu'ils doivent à présent capter les processus d'expérimentation par lesquels les institutions et les acteurs se construisent mutuellement. Kristensen et Morgan (2012) appellent d'ailleurs au développement d'une "théorie de la co-constitution des institutions et des acteurs» pour étudier la manière dont des acteurs hérités de la période fordiste se forgent de nouvelles identités, de nouveaux rôles organisationnels, voire des aspirations inusitées en se mettant en relation avec des institutions émergeantes à lère de la mondialisation productive. Dans cette optique, nous proposons de définir un cadre d'analyse novateur en procédant à une hybridation entre le courant de l'entreprenariat institutionnel et les approches des processus politiques et du cadrage pour étudier les stratégies déployées par des acteurs de la société civile qui souhaitent participer à l'institutionnalisation du champ de régulation de la RSE. Nous soutenons que cette combinaison théorique représente un fort potentiel pour conceptualiser les liens complexes entre l'acteur et la structure en situant les actions collectives dans le processus du changement institutionnel.

\section{Faire dialoguer la théorie néo-institutionnelle et la théorie des mouvements sociaux pour situer les actions collectives dans le processus du changement institutionnel}

Si les coalitions réunissant des acteurs de la société civile sont relativement peu étudiées dans la littérature, les défis qu'ils doivent surmonter dans ce processus d'expérimentation institutionnelle le sont encore moins. La plupart des travaux conduits sur les relations intra mouvements et inter mouvements s'intéressent le plus souvent à la structuration de ces réseaux inter-organisationnels suivant la nouvelle ramification des chaines globales de valeur ainsi quau répertoire d'actions collectives développé dans cette optique par des regroupements plus ou moins formels (ArmbrusterSandoval, 2005; Palpacuer, 2008; Hennebert, 2008; DufourPoirier, 2011; Anner, 2013). Ces travaux appréhendent généralement ces rapprochements dans l'hypothèse d'une transnationalisation des mouvements sociaux faisant abstraction des cadres institutionnels dans lesquels ils s'inscrivent. À contre-courant, Vercher (2009) s'intéresse par exemple à la production de cadres d'interprétation d'enjeux globaux par une coalition participant au mouvement transnational anti sweatshop pour établir son action dans un contexte institutionnel particulier. Chemin et Vercher (2011) exposent d'ailleurs plus avant les tensions expérimentées par ces acteurs pour lier l'action de la coalition à l'instrument de régulation qui incarne ses valeurs tout en maintenant une cohésion organisationnelle fragilisée par la présence de logiques institutionnelles hétérogènes. Si ces travaux rendent compte du travail d'articulation de l'action à la structure sociale, ils ne parviennent cependant pas à situer les acteurs au sein des rapports de force qui structurent le processus d'institutionnalisation de la RSE.

Dans notre perspective, nous envisageons la RSE comme un "champ d'action stratégique» (Fligstein et McAdam, 2012), c'est-à-dire un niveau intermédiaire d'action que l'on retrouve aussi dans la littérature sous le terme de «champ organisationnel» (DiMaggio et Powell, 1983), au sein duquel se situent différentes catégories d'acteurs en compétition, voire en conflit plus ou moins ouvertement. Au sein de ce champ d'action stratégique, les acteurs sont engagés dans ce que nous concevons comme une lutte institutionnelle qui porte sur la création de règles spécifiques et l'allocation des ressources (Fligstein et McAdam, 2012). Contrairement aux travaux fondateurs des néo-institutionnalistes qui éludaient le rôle du pouvoir et du conflit dans les processus d'institutionnalisation, nous considérons que c'est la constitution d'un rapport de force entre les acteurs sociaux, les dirigeants d'entreprise, et les actionnaires porteurs d'intérêts divergents, voire conflictuels, qui structure le champ d'action stratégique de la RSE.

La constitution d'une «coalition politique» est une stratégie couramment envisagée par des groupes d'acteurs qui cherchent à créer un rapport de force afin de participer au processus d'institutionnalisation et façonner la structuration d'un champ d'action stratégique (Fligstein et McAdam, 2012 : 14). Les rapprochements qui sopèrent plus ou moins formellement entre les acteurs de la société civile s'apparentent selon nous à une forme de coalition politique dont la singularité est de se composer d'acteurs organisationnels ayant des logiques institutionnelles plus ou moins hétérogènes. Par «logique institutionnelle», nous entendons un «ensemble des systèmes de croyance et de pratiques qui y sont associées et qui prédominent dans un champ organisationnel» (McAdam et Scott, $2005: 15)$. Une coalition d'acteurs de la société civile se compose généralement de représentants de syndicats, d'organismes de solidarité internationale et de développement international, d'organisations militant pour la protection de l'environnement, d'associations étudiantes, d'organisations pour la protection de l'enfance, des droits des femmes et des minorités qui sont porteurs de logiques institutionnelles distinctes, parfois conflictuelles.

Ainsi, nous soutenons que la participation d'une coalition multi acteurs à l'institutionnalisation du champ d'action stratégique de la RSE repose sur deux habilités sociales particulières. D'une part, une coalition doit savoir produire des «cadres culturels» capables d'enligner une pluralité d'acteurs organisationnels sur une action collective commune et leur conférer des rôles et des identités organisationnels, voire de nouvelles aspirations pour les inscrire au sein de ce champ d'action stratégique. D'autre part, elle doit savoir élaborer une série de stratégies visant à diffuser ses idées et les faire « encastrer» à différents niveaux institutionnels. 


\section{LA PRODUCTION DE CADRES CULTURELS}

Fligstein et McAdam expliquent que le déploiement d'une coalition réunissant une pluralité de logiques institutionnelles repose sur des «acteurs dotés d'habilités sociales» («Social Skilled Actors») particulières pour parvenir à faire collaborer, dans un but commun, des acteurs ayant des identités, des cultures et des aspirations différentes. Ces acteurs dotés d'habilités sociales distinctes - dont le rôle dans un champ organisationnel en émergence rejoint celui des « entrepreneurs institutionnels » tel que décrit par les néo-institutionnalistes (DiMaggio, 1988) - sont capables, à travers la production de "cadres culturels", de créer un sens commun à un champ d'action stratégique, proposer à ses membres une action fédératrice pour s'y déployer, et définir à chacun des rôles et des identités organisationnels les y faisant prendre place (Fligstein et McAdam, 2012).

S'inscrivant dans le courant de l'interactionnisme symbolique, les «cadres culturels» permettent de créer de nouvelles relations entre l'agence et la structure sociale et agissent telles des ressources habilitantes sur les acteurs pour transformer les institutions. Bien que le concept de cadre culturel s'inspire de celui de "processus de cadrage» introduit en sociologie par Snow et al. (1986), celui-ci s'en distingue puisqu'il permet de considérer le rôle des cadres d'interprétation de l'action collective sur la structuration d'un champ organisationnel: la production de cadres culturels induit en effet que les entrepreneurs institutionnels attribuent, en plus de créer un sens partagé par rapport à un champ organisationnel et d'aligner les différentes composantes institutionnelles d'une coalition sur une conduite stratégique commune, des rôles et des identités organisationnels aux acteurs les faisant évoluer vers des intérêts et des aspirations renouvelés.

Comment cet acteur doté d'habilités sociales singulières construit-il des cadres culturels? À cet égard, le courant de l'entreprenariat institutionnel est particulièrement éclairant. Bien que souvent envisagée comme un individu, un consensus se dessine actuellement pour considérer la figure de l'entrepreneur institutionnel également comme une organisation, voire un groupe d'organisations (Battilana et al., 2009). À partir de cette littérature, nous raisonnons donc par analogie en considérant une coalition multi acteurs comme un «entrepreneur institutionnel organisationnel». Cependant, nous n'endossons pas la conception répandue d'un entrepreneur institutionnel dépeint comme un acteur stratégique et rationnel. D'une part, nous considérons que cette représentation sous-estime les apprentissages collectifs induits par un champ en émergence où les connaissances sont par définition partielles, voire lacunaires (Acquier et Aggeri, 2008). D’autre part, l'intérêt d'un entrepreneur institutionnel ne peut être connu au préalable puisqu'il se construit à mesure que les connaissances se développent dans un champ organisationnel sous l'effet des interactions entre les acteurs (ibidem). Nous estimons que le principal objectif d'un entrepreneur institutionnel est de parvenir à faire collaborer, dans le but de faire émerger un champ d'action stratégique, des acteurs ayant des identités, des intérêts et des aspirations parfois divergents à travers la production d'un cadre culturel (Fligstein et McAdam, 2012).

La manière dont un entrepreneur institutionnel procède pour produire un cadre culturel consiste à réaliser un «bricolage institutionnel ", c'est-à-dire à générer de nouvelles idées, pratiques, règles ou technologies en combinant des éléments institutionnels préexistants dans différents règnes idéationnels (Campbell, 2004 : 101). Cette définition apparaît problématique, car elle induit que les acteurs construisent des idées, des pratiques, des techniques novatrices à partir d'éléments institutionnels préexistants dans le but stratégique d'en faciliter son cheminement institutionnel. Si l'on s'en tient à ce raisonnement, les mouvements sociaux seraient seulement des porteurs d'idées et de significations disponibles au préalable. Bien que cette conception s'en inspire, les travaux de Snow et al. (1986) et Benford et Snow (2012) postulent plutôt que les acteurs sociaux sont engagés dans «des activités de production de sens». Dans notre perspective, une coalition peut à la fois agir stratégiquement en construisant un cadre culturel qui propose un certain consensus entre des acteurs ayant des intérêts, des idéologies et des aspirations différentes, et être engagée dans des activités de production de sens lui conférant ainsi une certaine forme de «leadership stratégique» (Campbell, 2005 : 63) en matière de RSE.

En plus d'incarner un projet consensuel et novateur, ce cadre culturel doit engager les acteurs dans la structuration de ce champ d'action stratégique en leur attribuant des rôles, des statuts, des identités organisationnelles pour qu'ils s'y enracinent (Fligstein et McAdam, 2012). Kristensen et Morgan (2012) soutiennent d'ailleurs qu'un processus d'expérimentation institutionnelle s'accompagne d'une transformation des acteurs collectifs, dont la plupart sont hérités de la période fordiste et qui doivent, à ce titre, évoluer pour participer à la négociation du cadre institutionnel émergeant à l'ère de la mondialisation productive. En produisant des cadres culturels qui intègrent le renouvellement de la dimension identitaire des acteurs collectifs, une coalition saura les accompagner dans ce processus d'expérimentation en les incitant à œuvrer avec créativité et à cultiver des intérêts et des aspirations inusités à travers notamment leur mise en relation avec des acteurs ayant des logiques institutionnelles hétérogènes. Si les aspects identitaires apparaissent comme un élément sur lequel il est possible d'agir, le concept de «travail institutionnel» de Lawrence et Suddaby (2006) permet d'identifier des stratégies qui visent à faire "encastrer» les idées à plusieurs niveaux institutionnels.

\section{ÉLABORATION DE STRATÉGIES DE DIFFUSION AGISSANT À PLUSIEURS NIVEAUX INSTITUTIONNELS}

Contrairement aux travaux menés en sociologie des mouvements sociaux, la structure d'opportunités politiques ne sapplique pas dans la perspective néo-institutionnaliste uniquement à l'État puisqu'il est entendu que les acteurs engagés dans un processus de changement institutionnel doivent également transformer les normes sociales, les valeurs et les représentations sociales (Scott, 1995; Campbell, 2005; Lawrence et Suddaby, 2006). Pour s'assurer que son 
innovation soit adoptée, un entrepreneur institutionnel doit en effet déployer son action à différents niveaux de juridiction que Scott (1995:35) envisage comme les piliers « régulateur», «normatif» et «cognitif», et sur lesquels agissent un ensemble de forces qui procurent une stabilité et un sens aux comportements sociaux. Ainsi, le «pilier régulateur» qui repose sur des mécanismes coercitifs, contraint et régule les comportements sociaux par le biais de règles, de lois et de sanctions généralement régies par les États, mais qui peuvent également impliquer le marché et les consommateurs individuels et institutionnels. Le "pilier normatif» se base sur des normes et des valeurs qui définissent non seulement la légitimité des objectifs et des buts poursuivis par une organisation, mais aussi la manière dont ils doivent être atteints (ibidem). Enfin, le «pilier cognitif» fait référence au système de croyances, de cultures, d'identités, de représentations sociales qui peuvent faciliter ou entraver les transformations institutionnelles (ibidem).

Lawrence et Suddaby (2006) précisent les actions pouvant être menées par des acteurs engagés dans un processus de création institutionnelle. En ce qui concerne les actions ciblant le pilier régulateur, ils auraient la possibilité de formuler des plaidoyers pour acquérir un soutien politique et une modification des réglementations afin de favoriser la diffusion d'une innovation; définir un système de règles conférant un rôle, une identité et un statut aux membres d'un champ organisationnel et en établissant également ses frontières; et imaginer des structures de règles qui établissent des droits de propriété. Ces actions visent en particulier l'État que l'on interpelle pour établir des structures qui favoriseront l'émergence d'un champ organisationnel constitué autour d'une innovation particulière. Les acteurs auraient également la possibilité d'agir sur le pilier normatif en construisant des identités, en définissant des relations entre un acteur et le champ dans lequel il opère; en transformant les liens normatifs, c'est-à-dire en modifiant les fondements moraux et culturels de certaines pratiques; en construisant les connexions inter organisationnelles à travers lesquelles des pratiques deviennent normativement contraignantes, et en constituant un groupe de pression chargé de veiller à leur conformité. Enfin, les acteurs pourraient agir sur le pilier cognitif en cherchant à stimuler le mimétisme entre les acteurs en inscrivant, par exemple, de nouvelles pratiques dans la continuité de procédés, technologies et règles existants et pris pour acquis; en théorisant autour de ces pratiques, c'est-à-dire en développant des catégories abstraites et des chaines de causalité; enfin, en abreuvant les acteurs de connaissances et de compétences afin qu'ils s'approprient la nouvelle institution.

Dans notre perspective, une coalition devrait s'assurer de développer des actions collectives qui ciblent l'ensemble de ces dimensions institutionnelles pour accompagner les modifications structurelles qu'elle revendique et qui nécessitent souvent une transformation des identités, des intérêts et des aspirations des acteurs collectifs et des individus. Nous considérerons la structure (État, marché), les acteurs collectifs et les individus comme des niveaux complémentaires sur lesquels une coalition doit agir sans exception pour assurer une certaine cohérence entre les règles, les normes sociales et les valeurs.

Par ailleurs, l'inscription de l'entrepreneur institutionnel au sein d'un "réseau relationnel» - ce que Campbell (2005) qualifie d' "encastrement relationnel» - serait un facteur essentiel pour assurer la diffusion de ses idées. En effet, l'accès à un réseau relationnel dense permet à un entrepreneur institutionnel d'assurer la diffusion d'une innovation qui structurera éventuellement un champ d'action stratégique, grâce aux interactions créées avec d'autres acteurs ayant des rôles différenciés et complémentaires. McAdam et Scott (2005) distinguent d'ailleurs trois classes d'acteurs qui ont un effet direct sur la structuration d'un champ organisationnel : les dominants, c'est-à-dire les individus, les groupes et les organisations autours desquels les actions et les intérêts d'un champ ont tendance à tourner; les challengers, c'est-à-dire les individus, les groupes et les organisations qui cherchent à défier la position avantageuse des dominants et les caractéristiques structurelles et procédurales fondamentales d'un champ organisationnel; les unités de gouvernance, c'est-à-dire les unités organisationnelles qui exercent un pouvoir et une autorité au niveau d'un champ. Chaque champ appartenant à un environnement social plus vaste, il comprend des acteurs externes, c'est-à-dire des individus, des groupes et des organisations qui ne sont généralement pas reconnus comme des participants d'un champ précis, mais qui influencent, de manière indirecte, l'action qui s'y déroule. Il implique en outre des unités de gouvernance externes qui sont des structures d'autorité et de pouvoir opérant à une échelle sociétale plus large et offrant des opportunités d'action (ou au contraire la contraignant) au niveau d'un champ organisationnel.

Ainsi, une coalition multi acteurs qui cherche à participer au processus d'institutionnalisation de la RSE pourrait par exemple identifier les challengers susceptibles d'agir comme des alliés dans ce champ d'action stratégique, les dominants quelle pourrait approcher pour faire modifier, par leur intermédiaire, les normes sociales en vigueur, les unités de gouvernance auxquelles s'adresser pour faire adopter de nouvelles règles et modifier loctroi des ressources, les mouvements sociaux qui pourraient appuyer ses revendications en exerçant des moyens de pression complémentaires, etc. Cette liste de cibles non exhaustive dépeint succinctement le mode opératoire d'une coalition qui aurait établi une stratégie pour assurer l'encastrement relationnel de ses idées.

\section{Les défis inhérents à la participation de la société civile au processus d'institutionnalisation de la RSE}

Nous formulons ici une série d'hypothèses de recherche se rapportant aux défis auxquels une coalition multi acteurs pourrait se confronter en participant directement au processus d'institutionnalisation de la RSE. Nous avançons qu'ils découlent d'une part, de la structuration du champ d'action stratégique actuellement dominé par une conception managériale de la RSE. Celle-ci est susceptible de limiter les acteurs dans la négociation d'un cadre d'interprétation quant 
à leur rôle et leurs perspectives d'action. D'autre part, ils se rapportent aux acteurs eux-mêmes et aux difficultés qu'ils peuvent connaitre pour déployer une action collective sous la forme d'une coalition politique compte tenu de l'hétérogénéité de leur logique institutionnelle. Loin dêtre exhaustives, ces hypothèses qui découlent d'une mise en dialogue entre une littérature théorique portant sur la RSE, les mouvements sociaux, l'entreprenariat institutionnel et les conséquences organisationnelles du pluralisme institutionnel visent plutôt à appréhender la complexité des défis avec lesquels les acteurs doivent conjuguer une fois engagés dans un tel processus dexpérimentation institutionnelle.

La première hypothèse concerne la prédominance dans ce champ d'action stratégique de l'approche managériale de la RSE qui impose aux acteurs de la société civile de transcender cette conception pour proposer une approche institutionnaliste de la RSE à laquelle ils pourraient être associés pour sa mise en œuvre. Ainsi, l'approche institutionnaliste de la RSE, à laquelle adhèrent les ONG et les syndicats, préconise un renforcement des capacités institutionnelles pour encadrer les actions des entreprises à travers la structuration d'un dialogue social élargi à partir d'Accords-cadres internationaux (ACI) sur la RSE ou de codes de conduite d'approvisionnement multipartites (Seignour et Vercher, 2011). À l'opposé, l'approche managériale que les gestionnaires et les organisations patronales soutiennent, soutient plutôt qu'une entreprise est capable de s'autoréguler si elle adopte des actions individuelles et volontaires orientées vers les salariés et les sous-traitants pour favoriser le développement de chaînes globales de valeur respectueuses des droits de l'Homme et des normes environnementales (ibidem). Dans notre optique, cette dernière approche représente un «idéal-type» puisque l'analyse des faits démontre que même les initiatives présentées comme unilatérales sont souvent le produit d'interactions plurielles entre des ordres juridiques étatiques et non étatiques (Vallée et al., 2003) lorsque celles-ci ne sont pas élaborées en lien avec les principaux interlocuteurs de l'entreprise (Gendron, 2013). Si nous reconnaissons que les initiatives unilatérales s'enchâssent elles-aussi dans des cadres institutionnels et des réseaux relationnels, il existe néanmoins des degrés variables à leur institutionnalisation qui leur confèrent un caractère plus ou moins contraignant. À ce chapitre, Campbell (2007) identifie les composantes institutionnelles à travers lesquelles les comportements des entreprises sont «médiatisés» et "contrôlés»: la mise en œuvre d'une régulation étatique forte obtenue sur la base d'un consensus élargi, lexistence d'un système industriel d'autorégulation, la présence d'ONG, de mouvements sociaux, d'investisseurs institutionnels et de la presse capables de surveiller les entreprises, lexistence d'incitatifs institutionnalisés, la présence d'associations commerciales et d'employeurs et une forme de dialogue institutionnalisée entre les syndicats, les employeurs, les ONG, les groupes communautaires et autres parties prenantes. $\mathrm{Si}$ lopposition que nous créons entre l'approche managériale et l'approche institutionnaliste de la RSE rend bien compte du degré d'institutionnalisation plus ou moins élevé des initiatives de RSE, elle indique surtout que ce processus repose sur les interactions créées par les acteurs de la société civile (ibidem).

Hypothèse 1 : La prédominance de l'approche managériale de la RSE impose aux acteurs sociaux d'inscrire leurs actions au sein de cette conception dominante en se servant des instruments d'autorégulation comme des opportunités pour créer un rapport de force susceptible de l'orienter vers une approche institutionnaliste de la RSE à laquelle ils pourraient sassocier pour sa mise en œuvre.

Cependant, les cadres culturels produits par une coalition doivent participer à ce que nous concevons comme une lutte portant sur les modalités d'institutionnalisation de la RSE en décriant à la fois le modèle qui domine actuellement ce champ d'action stratégique, c'est-à-dire l'approche managériale de la RSE, et en faisant la promotion de l'approche institutionnaliste de la RSE. Pour ce faire, une coalition doit agir à deux niveaux : elle doit d'une part, traduire la RSE dans la logique institutionnelle de ses membres en l'ancrant aux droits de l'Homme, du travail et de l'environnement. D'autre part, elle doit procéder à un bricolage institutionnel à partir déléments existants dans son environnement, afin de proposer des moyens de mises en œuvre innovants et concrets à l'approche institutionnaliste de la RSE sous la forme de règles, de normes et de valeurs (Campbell, 2004, 2007).

Hypothèse no 2: Une coalition fera la preuve de son leadership au sein de ce champ d'action stratégique en proposant une vision alternative de la RSE qui sera soutenue par des moyens concrets de mise en cuvre.

Néanmoins, nous anticipons que le modèle économique néolibéral au sein duquel est profondément enracinée l'approche managériale de la RSE (Bartley, 2007) limite les perspectives d'action d'une coalition en raison de l'idéologie de certains acteurs sociaux. Alors que les interférences des idéologies sur la négociation d'un cadre d'interprétation à une action collective demeurent largement sous-estimées (Contamin, 2010), celles-ci pourraient bien obscurcir les perspectives d'action d'une coalition dans la mesure où certains acteurs ne sont pas capables d'imaginer de modèles alternatifs se cristallisant plutôt sur une critique radicale de l'approche managériale de la RSE et du modèle économique auquel elle est associée. L'existence au sein de la société civile de discours plus radicaux se focalisant sur les politiques économiques néo-libérales, les entreprises multinationales et les instruments d'autorégulation nous amène à nous interroger sur les possibles interférences des idéologies sur la négociation d'un sens partagé par les acteurs sociaux quant à leur action, leur rôle et leur identité organisationnels dans le champ d'action stratégique de la RSE.

Hypothèse de recherche no 3 : La prédominance de lapproche managériale de la RSE dans ce champ d'action stratégique limite la capacité d'une coalition à produire un cadre culturel susceptible de fédérer autour d'une action commune une diversité d'acteurs sociaux dont lidéologie de certains peut sêtre construite en réaction aux politiques économiques néo-libérales. 
La quatrième hypothèse porte sur l'encastrement relationnel (Campbell, 2005) d'une coalition qui peut être restreint par le fait que son travail d'entrepreneur de l'innovation sociale appréhende le changement social dans une logique réformiste. En situant son action au sein de la sphère institutionnelle, une coalition mise en effet sur la transformation des pratiques à plus ou moins long terme, en créant des contraintes normatives, en ouvrant le dialogue avec des acteurs dominants et des unités de gouvernance ou en participant à des consultations publiques. Cependant, une coalition doit également compter sur l'appui d'autres mouvements sociaux pour créer une pression normative supplémentaire (McAdam et Scott, 2005). Or, nous soulevons l'hypothèse que l'approche réformiste préconisée par une coalition limite sa capacité d'encastrement relationnel auprès d'autres acteurs de la société civile en faveur d'un changement social radical.

Hypothèse de recherche no 4: La logique réformiste dans laquelle s'inscrit une coalition agissant comme un entrepreneur de l'innovation sociale limite sa capacité d'encastrement relationnel auprès d'autres acteurs de la société civile en faveur d'un changement social radical.

Les deux prochaines hypothèses de recherche portent sur les caractéristiques structurelles d'une coalition et leurs effets sur la production de cadres culturels. La littérature établit que le bricolage institutionnel est plus novateur si un entrepreneur institutionnel se situe au sein d'un réseau social dense et riche puisqu'il est alors en position de générer de nouvelles idées, pratiques, règles ou technologies à partir d'éléments institutionnels véhiculés dans d’autres règnes idéationnels qu'il recompose sous une forme innovante avant de l'importer dans un champ particulier (Campbell, 2004). Ce procédé explique d'ailleurs l'effet amplificateur des collaborations entre des acteurs provenant de champs organisationnels hétérogènes engagés dans des processus de créations institutionnelles (Phillips et al., 2000; Lawrence et al., 2002). Alors qu'ils mobilisent des règles et des ressources préexistantes dans leur champ respectif pour résoudre collectivement des problèmes spécifiques, le pluralisme des acteurs assure donc une plus grande créativité à cette collaboration de par la diversité des pratiques, des savoir-faire et des valeurs qu'ils y introduisent.

Hypothèse de recherche no 5 : Le pluralisme des acteurs organisationnels impliqués au sein d'une coalition ONG syndicats contribue à enrichir les cadres culturels produits grâce à la richesse des pratiques, des savoir-faire et des valeurs qu'ils introduisent au sein de cette collaboration.

En outre, la richesse du bricolage institutionnel dépend des caractéristiques structurelles de son réseau relationnel en raison de l'accès que celui-ci lui procure à une diversité d'acteurs porteurs d'idées essentielles au cheminement de son projet institutionnel : les programmes/les décideurs, les cadrages/les cadreurs, les sentiments publics/les électeurs, et les paradigmes/les théoriciens (Campbell, 2004: 101). Concrètement, les décideurs s'appuient sur des programmes pour légitimer leur choix. Les théoriciens tels que les universitaires et les intellectuels, contribuent à lélaboration de paradigmes. Les cadreurs facilitent l'acceptation de nouvelles idées par le grand public, les électeurs, etc. et ils en développent également à l'instar des publicitaires, etc. Campbell situe l'entrepreneur institutionnel à la croisée de ces différents règnes idéationnels : c'est cette position qui lui permet de transférer des idées d'une sphère à l'autre. Il est ici question de consultants et de «think-tanks» L'entrepreneur institutionnel fait en quelque sorte émerger un consensus entre des acteurs déconnectés par ce travail de bricolage institutionnel qui consiste à recomposer des éléments institutionnels préexistants qui bénéficient donc d'une certaine acceptation sociale.

Hypothèse de recherche no 6: La présence dans son réseau relationnel d'une diversité d'acteurs porteurs d'idées essentielles au cheminement de son projet institutionnel favorise la production de cadres culturels fédérateurs.

Les trois hypothèses de recherche suivantes s'intéressent à la question des effets organisationnels du pluralisme institutionnel. Réunissant des représentants de mouvements sociaux divers qui sont porteurs de logiques institutionnelles distinctes, une coalition correspond à une organisation évoluant dans un environnement institutionnel pluraliste dont elle doit tenir compte pour établir son action (Chemin et Vercher, 2011). Kraatz et Block (2008) définissent l'influence du pluralisme institutionnel, non pas comme des forces extérieures qui exercent des demandes coercitives et normatives concurrentes sur une organisation, mais plutôt comme des «effets institutionnels constitutifs et idéationnels» qui auraient des implications significatives au niveau organisationnel (2008: 244). S'intéresser à la manière dont une organisation conjugue avec la multiplicité des identités institutionnelles qui la compose devient d'autant plus pertinent lorsque l'on considère que le pluralisme est une source éventuelle d'incohérences, d'instabilités, d'ambiguïtés quant aux objectifs organisationnels, voire même de conflits entre divers groupes (ibidem).

Selon Kraatz et Block, une organisation enchâssée dans des différents ordres normatifs et évoluant ainsi dans un environnement pluraliste serait capable de satisfaire les diverses demandes qu'elle reçoit de ses multiples composantes institutionnelles en développant une action qui sache transcender son appartenance à plusieurs catégories institutionnelles. Kraatz et Block identifient deux facteurs qui auraient un effet déterminant sur la capacité d'une organisation à définir une action fédératrice pour ses membres, soit sa légitimité et son système de gouvernance qui se construisent de manière particulière en contexte de pluralisme institutionnel.

Alors que les néo-institutionnalistes sont d'avis que les organisations acquièrent une légitimité en se conformant symboliquement aux normes culturelles et aux attentes sociales présentes dans leur environnement, cette explication devient toutefois problématique en contexte de pluralisme institutionnel. Selon Kraatz et Block, une organisation qui doit répondre à de multiples systèmes sociaux ayant des normes, des valeurs et des croyances distinctes, voire contradictoires, se définit une identité a priori, c'est-à-dire avant d'activer les 
règles et les scriptes institutionnelles appropriées (2008:247). Ainsi, pour acquérir une légitimité aux yeux de ses membres, une coalition multi acteurs en tant qu'organisation évoluant dans un environnement pluraliste doit se doter d'une identité globalisante qui soit capable de transcender les multiples identités et ordres normatifs qui la composent.

Hypothèse no 7: La légitimité d'une coalition découle de son habilité à se définir une identité organisationnelle globalisante, c'est-à-dire une logique institutionnelle distincte de celles de ses membres capable de les unir dans une action collective novatrice.

Par ailleurs, Kraatz et Block endossent la perspective de Selznick (1957) sur la définition d'une «institution organisationnelle» qu'il envisageait comme étant infusée par les valeurs de ses composantes et agissant tel un véhicule à travers lequel ces groupes poursuivent leurs aspirations et leurs idéaux. À mesure que l'organisation développe sa propre logique et acquiert l'habileté d'apporter une identité à ses membres, celle-ci accepte leurs identités et leurs logiques à part égale (252 :2008). Lorsqu'une organisation devient une institution capable d'abriter les différentes composantes institutionnelles qui la composent, celle-ci doit cependant compter sur des mécanismes de gouvernance susceptibles d'aplanir les tensions éventuelles. Ce système de gouvernance devrait idéalement permettre aux acteurs de dépasser leurs différends pour se concentrer sur les objectifs poursuivis par leur organisation, et non sur la satisfaction de leurs intérêts immédiats.

Nous avançons qu'une coalition doit se munir d'un système de gouvernance visant à maintenir et favoriser l'expression d'un certain pluralisme institutionnel afin qu'elle s'alimente de cette diversité d'identités, de normes, de valeurs et de croyances pour élaborer son action collective. Campbell (2005) parle d'ailleurs de la propension d'un entrepreneur institutionnel à former une «communauté d'identités» - par opposition à une "communauté d'intérêts" - les pratiques, les savoir-faire et les valeurs étant mis à contribution pour définir une action collective innovante. Des mécanismes visant à assurer un certain équilibre entre les différentes composantes d'une coalition et faciliter l'expression et la prise en compte des différents points de vue lui permettront de mieux composer avec les tensions internes qui fragilisent sa cohésion. En effet, ce système de gouvernance devrait notamment développer ses habilités sociales à proposer des actions entrant en résonnance avec les valeurs et les attentes sociales de ses différentes composantes institutionnelles tout en incarnant une forme de leadership stratégique en matière de RSE susceptible de les faire évoluer dans leur rôle et leur identité, leurs aspirations et leurs intérêts.

Hypothèse no 8: Une coalition ONG - syndicats doit se munir d'un système de gouvernance afin de maintenir et favoriser l'expression d'un certain pluralisme institutionnel orientée vers la définition d'une action collective novatrice.

Cette éventualité constituant le scénario idéal, Kraatz et Block en identifient d'autres par lesquels une organisation pourrait se doter d'une légitimité dans un contexte de pluralisme institutionnel, soit en cherchant à éliminer le pluralisme, c'est-à-dire en marginalisant ou en évinçant certaines identités institutionnelles et obligations que leurs composantes veulent imposer; soit en se mettant en relation de manière indépendante, à un niveau symbolique, avec plusieurs composantes institutionnelles (2008:250).

Hypothèse no 9: Si la coalition ne parvient pas à imposer sa propre logique institutionnelle en offrant à ses membres une identité globalisante, elle sera susceptible de mettre en cuvre des stratégies visant à éliminer le pluralisme, soit en marginalisant des groupes, voire en les écartant, soit en se mettant en relation à un niveau symbolique avec certains faute de pouvoir enrayer les différends entre ses membres.

La dernière hypothèse de recherche concerne la question de la répartition du pouvoir au sein d'une coalition et du contrôle de ses ressources organisationnelles. Tattersall (2006) souligne à cet égard qu'une coalition doit s'assurer de disposer de certaines ressources organisationnelles pour garantir son autonomie par rapport à ses membres (i.e. un bureau officiel et indépendant, des sources de financements diversifiées, etc.). Vercher (2009) en fait d'ailleurs une condition essentielle pour qu'une coalition acquière son autonomie décisionnelle sans risquer de se voir imposer une logique institutionnelle par un de ses membres.

Hypothèse no 10: Le contrôle des ressources organisationnelles par une de ses composantes limite l'autonomie d'une coalition et sa capacité à se constituer une identité globalisante dans la mesure où celle-ci impose sa logique institutionnelle au regroupement.

\section{Conclusion}

L'objectif de cet article était de déterminer les défis inhérents à la participation directe des acteurs de la société civile à l'institutionnalisation du champ de régulation de la RSE. Pour ce faire, nous avons défini un cadre d'analyse combinant le courant de l'entrepreneuriat institutionnel et les approches des processus politiques et du cadrage afin de concevoir les actions qu'ils peuvent mener pour participer à ce que nous concevons comme une lutte portant sur les modalités d'institutionnalisation de la RSE. Nous avons considéré plus spécifiquement une stratégie couramment mise en œuvre par des acteurs qui cherchent à acquérir une certaine influence sur la structuration d'un champ stratégique d'action, soit la constitution d'une "coalition politique» (Fligstein et McAdam, 2012) réunissant des acteurs de la société civile. Nous avons établi que cette forme de regroupement doit développer deux habilités sociales particulières pour participer au processus d'institutionnalisation de la RSE. D'une part, elle doit savoir produire des cadres culturels qui enracinent les acteurs collectifs au sein de ce champ d'action stratégique en leur conférant des rôles, des statuts et des identités organisationnelles (Kristensen et Morgan, 2012; Fligstein et McAdam, 2012). D'autre part, elle doit savoir élaborer des stratégies de diffusion pour faire encastrer ses idées à plusieurs niveaux institutionnels que nous considérons complémentaires (coercitif, normatif, cognitif, relationnel) (Scott, 1995; Campbell, 2004, 2005). Sans se vouloir exhaustive, nous avons proposé une série de dix 
hypothèses pour saisir en un regard les tensions rencontrées par les acteurs sociaux une fois engagés dans ce processus. Celles-ci se rapportent d'une part à la structuration du champ d'action stratégique et à la capacité d'une coalition à produire un cadrage incarnant une conception institutionnaliste de la RSE et d'autre part, aux acteurs eux-mêmes qui sont porteurs de logiques institutionnelles parfois opposées à celle que la coalition doit développer pour participer au processus d'institutionnalisation de ce champ d'action stratégique. Cette dissonance que nous appréhendons au niveau des logiques institutionnelles appelle selon nous à des recherches empiriques pour explorer l'existence de possibles distorsions cognitives chez certains acteurs sociaux entre leurs représentations d'une action collective traditionnelle et celles qu'ils se construisent de leur travail réalisé en tant qu'entrepreneur de l'innovation sociale.

\section{Bibliographie}

Acquier, Aurélien; Aggeri Franck (2008). «Entrepreneuriat institutionnel et apprentissages collectifs. Le cas de la Global Reporting Initiative», Management International, vol. 12, no. 2, p. $65-80$.

Anner, Mark (2013). «Workers'Power in Global Value Chains : Fighting Sweatshop, Practices at Russel, Nike and Knights Apparel ", dans P. Fairbrother, C. Lévesques et M-A Hennebert (sous la direction de), Transnational Trade Unionism : Building Union Power, Routledge, p. 23-41.

Armbruster-SAndoval, Ralph. (2005). «Workers of the World Unite? The Contemporary Anti-Sweatshop Movement and the Struggle for Social Justice in the Americas». Work and Occupations, vol. 32, no. 4, p. 464-485.

Audet, René. (2013). «Certifications : la construction d'un cadre régulatoire hybride», dans C. Gendron et B. Girard, Repenser la responsabilité sociale de l'entreprise, Armand Colins, p. 167181.

BARtley, Tim (2007). "Institutional Emergence in the Era of Globalization : The Rise of Transnational Private Regulation of Labor and Environmental Conditions», American Journal of Sociology, vol. 113, no 2, p. 297-351.

Battillana, Julie; Bernard Leca; Boxembaum Eva (2009). «2 How Actors Change Institutions : Towards A Theory of Institutional Entrepreneurship ", The Academy of Management Annals, vol. 3, no 1, p. 65-107.

BЕск, U. (1986). La société du risque : sur la voie d'une autre modernité, Paris, Alto Aubier, 521p.

Benford, Robert D.; Snow David A. (2012). «Processus de cadrage et mouvements sociaux : présentation et bilan", Politix, vol. 3n, no 99, p. 217-255.

CAmpbell John L. (2007). «Why Should Corporations Behave in Socially Responsible Ways? An Institutional Theory of Corporate Social Responsibility", Academy of Management Review, vol. 32, no 3, p. 946-967.

Campbell John L. (2005). "Where Do We Stand? Common Mechanisms in Organizations and Social Movements Research », dans G. F. Davis, D. McAdam, W. Richard, S. Mayer et N. Zald (sous la direction de), Social Movements and Organizational Theory, p. 41-68.
Campbell, J. L. (2004). Institutional Change and Globalization, Oxfordshire, Princeton University Press, 247 p.

Chemin, Carine; Vercher Corinne (2011). "The Challenge of Activist Coalition Governance : Accommodating Diversity to Create Institutions - An approach Via the Inter-relationships Between Action, Project and Instrument», Volontas, no 22, p. 682-705.

Contamin, Jean-Gabriel (2010). «Cadrages et luttes de sens». dans E. Agrikoliansky, I. Sommier et O. Fillieule (sous la direction), Penser les mouvements sociaux, La Découverte «Recherches», p. 55-75.

DiMaggio, Paul. J. (1988). «Interest and Agency in Institutional Theory», dans L. G. Zucker, Institutional Patterns and Organizations : Culture and Environment, Ballinger, p. 3-21.

DiMaggio, Paul. J.; Powell Walter W. (1983). «The Iron Cage Revisited : Institutional Isomorphism and Collective Rationality in Organizational Fields", American Sociological Review, Vol. 48, p. 147-160.

Dufour-Poirier, M. (2011). "Construction d'une coalition syndicale internationale : analyse d'une perspective Nord-Sud», Thèse de doctorat, HEC-Montréal, $520 \mathrm{p}$.

Fligstein, Neil; McAdam, Doug (2012). A Theory of Fields, New York, Oxford Publishing University, 256 p.

Gendron, Corinne (2001). «Émergence de nouveaux mouvements sociaux économiques», Paris : Revue Pour, No 172, décembre 2001, p. 175-181

Gendron, Corinne; Lapointe Alain; Turcotte Marie-France (2004). «Responsabilité sociale et régulation de l'entreprise mondialisée». Relations industrielles, vol. 59, nº 1, p. 73-100.

Gendron, Corinne (2013). "Les initiatives et les dispositifs de responsabilité sociale menacent-ils le droit international : réflexions à partir d'ISO 26000 en matière de droit du travail», dans D. Brunelle et J. Baker (dir.). Travail et commerce : clauses sociales, responsabilité sociale et accords transnationaux d'entreprise, Éditions IEIM, p. 55-92.

Gereffi, Gary (2001). «Beyond the producer-driven/buyerdriven dichotomy : the evolution of global value chains in the internet era». IDS Bulletin, vol. 32, no 3, p. 30-39.

Hennebert, M-A. (2008). "De la transnationalisation de l'action syndicale au sein des entreprises transnationales : une analyse de «Réseau UNI@Quebecor World»». Thèse de doctorat, Montréal, Université de Montréal, 650 p.

Kolk, Ans; Tulder Rob Van (2005). "Setting new global rules? TNCs and codes of conduct», Transnational Corporations, vol. 14 , no. 3, p. 1-28.

KraAtz, Matthew S.; Block, Emily (2008). «Organizational implications of institutional pluralism», dans R. Greenwood, C. Oliver, R. Suddaby et K. Sahlin (sous la direction de.), The SAGE handbook of organizational institutionalism., SAGE Publications, p. 243-276.

IgALEns, Jacques (2013) «Corégulation, l'expérience européenne», dans C. Gendron et B. Girard, Repenser la responsabilité sociale de l'entrepris, Armand Colins, p. 167-181.

Kristensen Peer Hull; Morgan Glen (2012). «From Institutional Change to Experimentalist Institutions", Industrial Relations, vol. 50, no S1, p. 413-436. 
Lawrence, Thomas B.; Suddaby Roy (2006). "Institutions and Institutional Work» dans S. R. Clegg, C. Hardy, T. B. Lawrence et W. R. Nord (sous la direction de), Handbook of Organization Studies, Sage, p. 215-254.

Lawrence, Thomas B.; Cynthia Hardy; Nelson Philipps (2002). «Institutional Effects of Interorganizational Collaboration : The Emergence of Proto-Institutions ». The Academy of Management Journal, vol. 45, no 1, p. 281-290.

McAdAm, Doug; Scott W. Richard (2005). «Organizations and Movements», dans G. F. Davis, D. McAdam; W. Richard, S. Mayer et N. Zald (sous la direction de), Social Movements and Organizational Theory, p. 4-41.

Micheletti, Michele (2003). "Global (Sub)Political Representation : The Clean Clothes Campaign and No Sweat Movement », Document de travail pour le ECPR Joint Sessions, Atelier no 14 «Political Representation», Edinburgh.

O’Rourke, Dara (2003). «Outsourcing Regulation : Analyzing Nongovernmental Systems of Labor Standards and Monitoring», The Policy Studies Journal, vol. 31, no. 1, p.1-31.

PAlpacuer, Florence (2008). «Firme-réseau globale et réseaux transnationaux d'ONG : Vers un nouveau mode de régulation?», Revue de la régulation, no 2, p. 1-16

Philipps, Nelson; Lawrence Thomas B.; Hardy (2000). «Interorganizational Collaboration and the Dynamics of Institutional Field", Journal of Management Studies, vol. 37, no 1, p. 23-43.

Scotт, Richard W. (1995). Institutions and Organizations, Thousand Oaks, London, New Delhi : Sage Publications, 178 p.

Seignour, Amélie et Corinne Vercher (2011). «Les Accords cadres internationaux faces au challenge de la régulation sociale des chaînes globales de valeurs", Revue de l'organisation responsable, no 1 , vol. 6 , p. 38-54.
SELZNICK, Philips. 1957. Leadership in administration : A sociological interpretation, Berkeley : University of California Press, $162 \mathrm{p}$.

Snow, David A.; E. Burke Rochford, Jr.; Steven K. Worden; Benford Robert D. (1986). "Frame Alignment Processes, Micromobilization, and Movement Participation", American Sociological Review, vol. 51, no. 4, p. 464-481.

Sobczak, André (2008). «Syndicats et responsabilité sociale des multinationales ", Gestion : Revue Internationale de Gestion, vol. 33 , no 1 , p. 18-26.

Sobczak, André (2004). «La responsabilité sociale de l’entreprise : Menace ou opportunité pour le droit du travail?», Relations industrielles, vol. $59, \mathrm{n}^{\circ} 1$, p. 26-51.

Tattersall, Amanda (2006). «Labor-Community Coalitions, Global UniZn Alliances, And the Potential of SEIU's Global Partnerships». In Global Unions : Challenging Transnational Capital Through Cross-Border Campaigns sous la dir. de Kate Bronfenbrenner, Cornell University Press, p. 155-173.

Utting, Peter (2001). Regulating Business Via Multistakeholder Initiatives : A Preliminary Assessment, Genève : United Nations Research Institute for Social Development, $43 \mathrm{p}$.

Vallée, Guylaine; Murray, Gregor; Coutu, Michel; Rocher, Guy et Giles Anthony (2003). Les codes de conduite des entreprises multinationales canadiennes : aux confins de la régulation privée et des politiques publiques du travail, Recherche subventionnée par le Conseil de recherche en sciences humaines du Canada et la Commission du droit du Canada dans le cadre du Programme conjoint sur les rapports en évolution, $110 \mathrm{p}$.

VERCHER, Corinne (2009). «La capacité régulatoire des initiatives multi-partites comme élément explicatif de lémergence et de la pérennité de l'action collective. Le cas du collectif français «Éthique sur l'étiquette» ». 4ème Congrès du RIODD, Lille. 